\title{
Empirical Modeling of a Heat Transfer Process using Local Models and Interpolation
}

\author{
Tor A. Johansen $\nmid$ and Bjarne A. Foss $\ddagger$ \\ $\dagger$ SINTEF Automatic Control, N-7034 Trondheim, Norway. \\ $\ddagger$ Department of Engineering Cybernetics, Norwegian Institute of Technology, \\ University of Trondheim, N-7034 Trondheim, Norway.
}

\begin{abstract}
Identification and control experiments were performed on a laboratory scale heat transfer process. An NARX model based on a combination of several local ARX models is identified. This model is compared to a simple ARX model and a non-linear mechanistic model, both for prediction and model predictive control.
\end{abstract}

\section{Introduction}

In science and engineering, there are two basic approaches to modeling, the empirical and the mechanistic approaches. The mechanistic approach is characterized by i) An understanding of the underlying physical mechanisms that governs the system's behavior is needed. ii) It is often very time-consuming. iii) The model is often valid for a wide range of operating conditions. iv) The model (and the modeling process itself) contributes to our understanding of the phenomena being studied. v) The model representation is compatible with many engineers understanding of the system behavior, and allows easy interpretation. vi) The model may form a basis for a wide range of purposes. The empirical approach, on the other hand, is characterized by i) A large amount of empirical data from all interesting operating conditions is needed. ii) The model may give very accurate predictions when applied under similar operating conditions as the data were logged under, but may give very inaccurate predictions when applied under different operating conditions. iii) One does not need to understand the system mechanisms. iv) The model is often difficult to validate, interpret, and analyze. v) The applicability of the model is often restricted to prediction and control.

Hence, when choosing a modeling framework, there are several benefits and disadvantages that must be weighted against each-other. In this paper we will try to illustrate some of these aspects, with emphasize on models developed for model based control purposes. In particular, we will compare a model developed using the semi-empirical non-linear modeling framework described in $[1,2]$ with models developed using more traditional methods, namely an ARX model and a mechanistic model.
The non-linear modeling framework described in $[1,2]$ is based on a decomposition of the system's operating range into a number of operating regimes, and the use of local models to describe the system's behavior within each operating regime. The local models are combined using an interpolation procedure, based on smooth weight functions. The decomposition into regimes is based on model variables that characterizes the system's different modes of behavior. More details and other examples can be found in $[1,2,3]$.

\section{Identification results}

Consider the experimental setup illustrated in Fig. 1. The output $y(t)$ of this system is given by a temperature sensor, the input is the voltage over the resistor $u(t) \in[0,10]$. In addition, the valve opening angle $v(t) \in\left[0^{\circ}, 180^{\circ}\right]$ is an independent measured variable that can be manipulated by hand only.

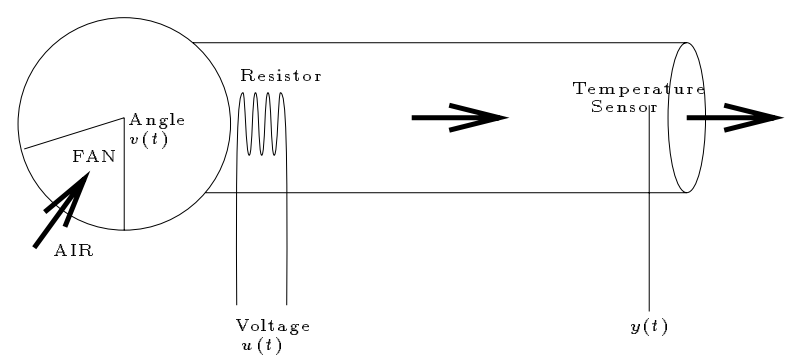

Figure 1: Heat Tube: Air is pulled into the tube by a fan through a valve. The air is heated by a resistor, and the air temperature is measured at the outlet. The length of the tube is about $30 \mathrm{~cm}$.

On this system the following experiments have been performed. First, consider the responses to a 10 volt step input, for $v_{1}=20^{\circ}, v_{2}=50^{\circ}$, and $v_{3}=100^{\circ}$ plotted in Fig. 2a. From these curves, we make two observations.

First, there seems to be two dominating timeconstants, at least for small valve opening angles $v$. 

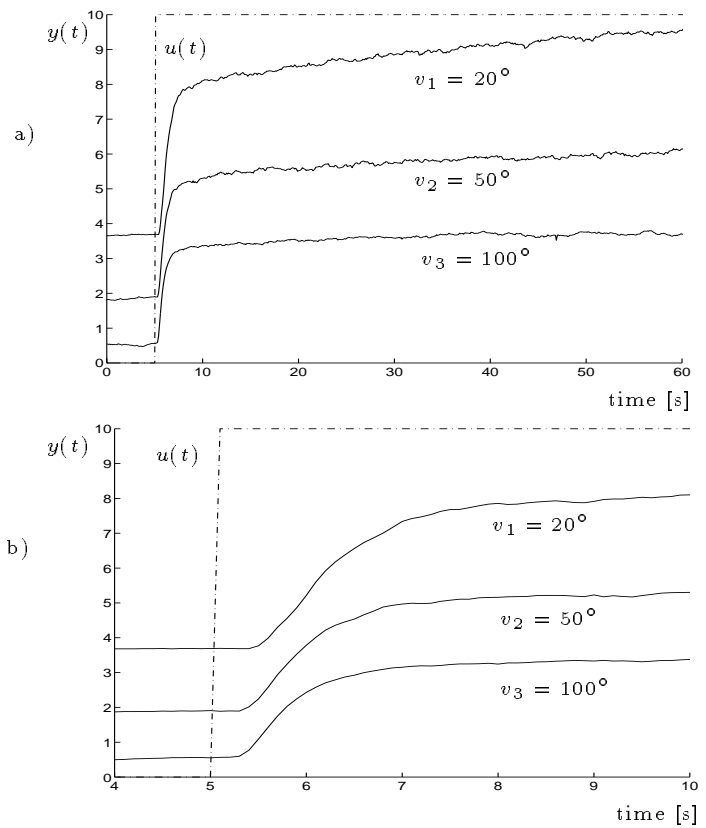

Figure 2: a) Response to a 10 volt step input, and b) the same response on a shorter timescale.

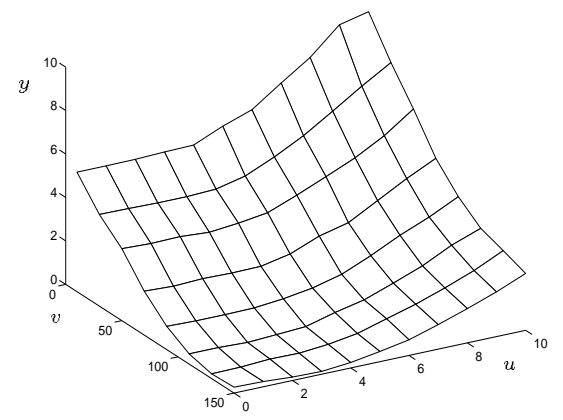

Figure 3: Steady-state response.

The fast mode (about 1 second) is related to the heat capacity of the air in the tube, while the slower one (a few minutes) is related to the heat capacity of the tube and the rest of the equipment. In the proceeding modeling and identification experiments, we will only attempt to find a model with good prediction performance on the horizon of the shortest of these time-constants, as this is the one that is interesting for typical control purposes.

Second, the steady-state gain seems to be a functions of the valve opening. On a shorter time-scale, cf. Fig. 2b, we see that also the time-constant and timedelay are functions of $v$. Similar experiments with fixed $v$ but varying input step size indicates that the steady-state gain, time-constant, and time-delay are functions of $u$, too, $\mathrm{cf}$. the steady-state response in Fig. 3.
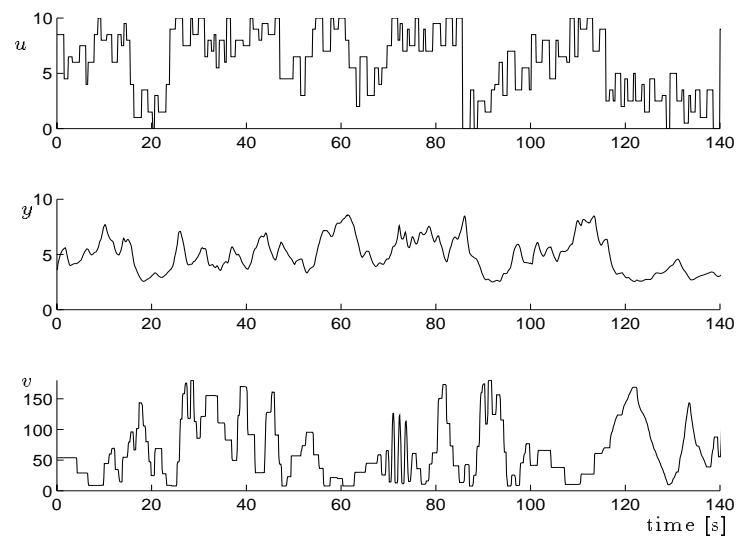

Figure 4: Time-series used for identification.

For the purpose of identification, we use the data sequence in Fig 4. The sampling interval is $\Delta t=0.11 \mathrm{~s}$, and the sequence contains about 1300 samples. The input $u(t)$ is a random signal exciting the system about a sequence of random operating points. The valve opening $v(t)$ varies over the full range of operation in a random manner. For the purpose of model validation, we use another data sequence with somewhat different excitation signals. The input varies in a qualitatively similar manner, while the valve opening angle varies more or less systematically to cover a wide range of frequencies and levels.

\subsection{Identification of ARX model}

Using the identification data sequence and the least squares algorithm, we find the following first-order ARX model

$$
\begin{aligned}
y(t)= & 0.2247+0.9284 y(t-1)+ \\
& 0.0352 u(t-4)-1.286 \cdot 10^{-3} v(t-5)
\end{aligned}
$$

The order and time-delays are determined from the step-responses, cf. Fig. 2 b.

\subsection{Identification of non-linear model based on local models}

From the discussion above, it is clear that the coefficients of the ARX model (1) should be functions of both $u$ and $v$. On the basis of the step-responses and the steady-state response, cf. Figs. 2 and 3, we therefore try to combine 4 local ARX model structures into an NARX model structure as described by [2]. The idea is to decompose the system's full range of operation into a set of operating regimes with a local ARX model that describes the system behavior within each regime. The 4 local ARX models are given relative weight in the interpolation of the local models as illustrated in Fig. 5. These weights are chosen to give a reasonable overlap. It is our experience that the exact shape and amount of overlap is of minor importance. The motivation behind this particular decomposition is that we believe a linear ARX model gives an adequate description of the system within each regime. Using the least squares algorithm and a cost index that penalizes mismatch between the global model 


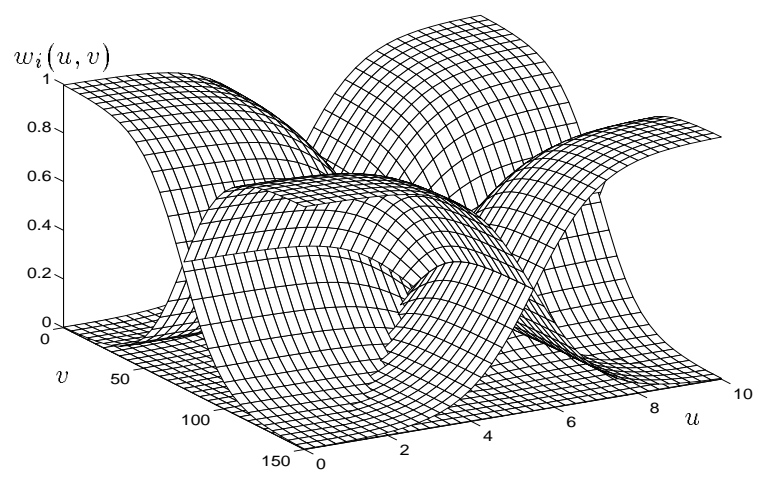

Figure 5: Weight functions for the local models.

prediction and the data gives the model

$$
\begin{aligned}
y(t)= & (0.3338+0.9196 y(t-1)+0.0049 u(t-4) \\
& \left.-1.411 \cdot 10^{-3} v(t-5)\right) \cdot w_{1}(u(t-4), v(t-5)) \\
& +(0.2029+0.9368 y(t-1)+0.0397 u(t-4) \\
& \left.-2.389 \cdot 10^{-3} v(t-5)\right) \cdot w_{2}(u(t-4), v(t-5)) \\
& +(0.5433+0.8347 y(t-1)+0.0139 u(t-3) \\
& \left.-0.946 \cdot 10^{-3} v(t-5)\right) \cdot w_{3}(u(t-4), v(t-5)) \\
& +(0.5181+0.8601 y(t-1)+0.0522 u(t-3) \\
& \left.-2.135 \cdot 10^{-3} v(t-5)\right) \cdot w_{4}(u(t-4), v(t-5))
\end{aligned}
$$

which we will denote "the 4-local model". The timedelays are chosen to be smaller at large valve opening angle, cf. Fig. 2b. We see that the local models have captured the facts that the dynamics is faster at large valve-opening, and that the gain is larger at high input voltages.

2.3. Identification of a mechanistic model A simple energy balance for this system is

$$
\begin{array}{r}
\rho V c_{p} \frac{d}{d t} T(t)=\rho c_{p} q\left(v\left(t-\tau_{v}\right)\right)\left(T_{0}-T(t)\right) \\
+U\left(T_{1}-T(t)\right)+G u^{2}\left(t-\tau_{u}\right)+Q
\end{array}
$$

where the symbols are defined in Table 1 . The volumetric air flow rate $q$ as a function of the valve opening angle $v$ depends on the fluid dynamics in the fan housing. We choose the empirical correlation $q(t)=k v(t)$, as a higher order correlation does not appear to give a model with significantly better prediction performance. In addition, the following measurement equation is determined experimentally

$$
y(t)=0.8173(T(t)-26.6)
$$

where $y(t)$ is the output voltage of the temperature sensor. Again, we identify the unknown physical parameters using the least squares algorithm, which gives $\hat{G}=0.0318 \Omega^{-1}, \hat{U}=0.5616 \mathrm{~J} / \mathrm{Ks}$, $\hat{k}=1.4920 \cdot 10^{-3} \mathrm{l} / \mathrm{s}$, and $\hat{Q}=2.0346 \mathrm{~W}$. Discretizing the model (3) using the identified parameters, we may write this model on the NARX form

$$
\begin{aligned}
& y(t)=0.3028+0.9265 y(t-1)+0.540 \cdot 10^{-3} v(t-5) \\
& -0.234 \cdot 10^{-3} y(t-1) v(t-5)+2.401 \cdot 10^{-3} u^{2}(t-4)
\end{aligned}
$$

The design of semi-mechanistic models of this form is discussed in [4].

\begin{tabular}{llll}
\hline & Variable & Value & Unit \\
\hline$v$ & Valve opening & & ${ }^{\circ}$ \\
$\rho$ & Mass density for air & 1.2 & $\mathrm{~g} / \mathrm{l}$ \\
$V$ & Volume of tube & 0.7 & $\mathrm{l}$ \\
$c_{p}$ & Air spec. heat cap. & 1.0 & $\mathrm{~J} / \mathrm{g} \mathrm{K}$ \\
$q$ & Volumetric air flow & & $\mathrm{l} / \mathrm{s}$ \\
$k$ & "Fan coefficient" & & $1 / \mathrm{s}$ \\
$G$ & Resistor conductance & & $\Omega^{-1}$ \\
$U$ & Heat transfer coeff. & & $\mathrm{J} / \mathrm{K} \mathrm{s}$ \\
$Q$ & Heat through fan & & $\mathrm{W}$ \\
$T$ & Tube air temp. & & ${ }^{\circ} \mathrm{C}$ \\
$T_{0}$ & Envir. air temp. & 24 & ${ }^{\circ} \mathrm{C}$ \\
$T_{1}$ & Equip. temp. & 28 & ${ }^{\circ} \mathrm{C}$ \\
$\tau_{v}$ & Time-delay & 0.55 & $\mathrm{~s}$ \\
$\tau_{u}$ & Time-delay & 0.44 & $\mathrm{~s}$ \\
\hline
\end{tabular}

Table 1: Symbols, constants and variables used in the mechanistic model.

\subsection{Discussion of identification results}

The prediction performance of the three identified models are compared by simulations on the validation data, cf. Fig. 6. It is evident that both the empirical 4-local and mechanistic models perform better than the ARX model, as one should expect. However, there does not appear to be any significant difference between the 4-local and mechanistic models. Notice that because the validation data were logged on a day with higher environment temperature, there was a significant, but constant, offset between the output of the simulation and the logged data. As this is easily compensated for with integral action in a controller, this offset was removed in Fig. 6 to simplify the comparison.

\begin{tabular}{lll}
\hline Model & $\begin{array}{l}\text { 1-step } \\
\text { pred. error }\end{array}$ & $\begin{array}{l}\text { 10-steps } \\
\text { pred. error }\end{array}$ \\
\hline ARX & 0.0788 & 0.4669 \\
4-local & 0.0787 & 0.3447 \\
Semi-physical & 0.0654 & 0.3182 \\
\hline
\end{tabular}

Table 2: Estimated average prediction errors, where a) is with the ARX model, b) is with the 4local mode, and $\mathrm{c}$ ) is with the mechanistic model.

The average 1-step-ahead and 10-steps-ahead prediction errors are estimated using the validation data sequence for the three models are shown in Table 2 . We see that the mechanistic model performs somewhat better than the 4-local model. The 10-stepsahead prediction errors reveal that there is a significant amount of unmodeled dynamics left. This may 

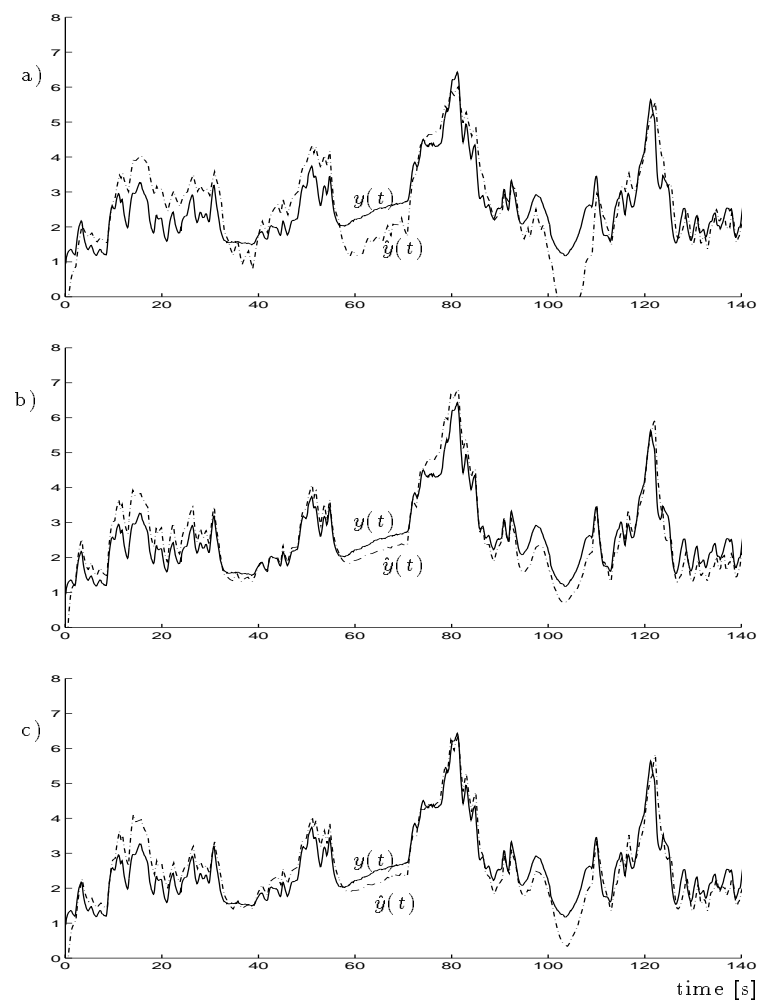

Figure 6: Simulation of the identified models on the validation data. a) ARX model, b) 4-local model, and c) mechanistic model.

be due to the slow time-constant we have neglected and other effects that are not adequately modeled, as well as the fact that the one-step-ahead prediction error criterion need not lead to a model with good multi-step prediction performance.

\section{Model predictive control (MPC) results}

For control purposes, we suggest a model of the following form

$$
\begin{aligned}
& y(t)=f(y(t-1), u(t-4), v(t-5))+e(t) \\
& e(t)=e(t-1)+\zeta(t)
\end{aligned}
$$

where $\zeta(t)$ is white noise, and $f$ is given by any of the identified models described in the previous section. This model includes the slowly time-varying disturbances and slow unmodeled dynamics, and automatically gives integral action when applied for MPC $[5,6]$. On the basis of this model, we formulate an on-line multi-step-ahead predictor where the bias is estimated by

$$
\hat{e}(t)=\hat{e}(t-1)+K(y(t)-\hat{y}(t \mid t-1))
$$

where the constant $K=0.003$ may be viewed as a "Kalman-filter gain". This value is chosen in an ad. hoc. manner in order to get a good trade-off between fast integral action and robustness. The input is parameterized as follows

$$
u(t+\tau)= \begin{cases}\theta_{1}, & \text { if } \tau \in\{0,1\} \\ \theta_{2}, & \text { if } \tau \in\{2,3,4\} \\ \theta_{3}, & \text { if } \tau \geq 5\end{cases}
$$

The control input is chosen as the first part of the control sequence minimizing the criterion

$$
\begin{gathered}
J\left(t, \theta_{1}, \theta_{2}, \theta_{3}\right)=\frac{1}{T_{h}} \sum_{\tau=1}^{T_{h}}\left(y^{\star}(t)-\hat{y}\left(t+\tau \mid t, \theta_{1}, \theta_{2}, \theta_{3}\right)\right)^{2} \\
+\lambda\left(\left(u(t-1)-\theta_{1}\right)^{2}+\left(\theta_{1}-\theta_{2}\right)^{2}+\left(\theta_{2}-\theta_{3}\right)^{2}\right)
\end{gathered}
$$

at every sampling instant. We have chosen the prediction horizon $T_{h}=14$ samples, which is about equal to the system's time-constant, and $\lambda=1.0$ for all control experiments. In other words, only the models are different in the experiments. The optimization problem is a non-linear programming problem, that is solved using Powell's direction set algorithm [7]. The search is initialized with the optimal control input from the previous step. The control algorithm is run on a 486 $\mathrm{PC}$, and to meet the real-time requirement, the search algorithm is allowed to run for at most 5 iterations, which is sufficient to give a close-to-optimal solution. No convergence problems were observed.

Tracking of a reference signal $y^{\star}$ is illustrated in Fig. 7 , while rejection of "disturbances" $v$ is illustrated in Fig. 8. We see that the disturbance rejection properties of the 3 controllers are essentially the same, while the controller based on the 4-local model is somewhat better for tracking than the others. This may be somewhat surprising, since the mechanistic model appeared to give better predictions, cf. Table 2. However, the introduction of a noise model has apparently improved the prediction accuracy of the 4-local model somewhat more than the other models.

\section{Concluding Remarks}

Based on our earlier experience with models based on local models, and this investigation, we can make the following comments on the relationship between the mechanistic and 4-local model. i) Both are relatively simple and give comparable prediction performance. The reason for this may be that our physical understanding of this simple system is quite good, and we have available a complete set of empirical data that covers all interesting modes of the system behavior. ii) The model predictive controllers based on the three models give comparable results. The conclusion must be that the non-linearities are so weak that they are not significant for control design. iii) The mechanistic model is based on an energy balance. The development of this model was quite straightforward, as the system is simple and most parts of it is well understood. The empirical model is based on empirical knowledge gained through experiments, and the development of this model was quite straigthforward as well. iv) The interpretability of both models are quite good. The mechanistic one obviously reflects the deepest understanding about what is going on in 

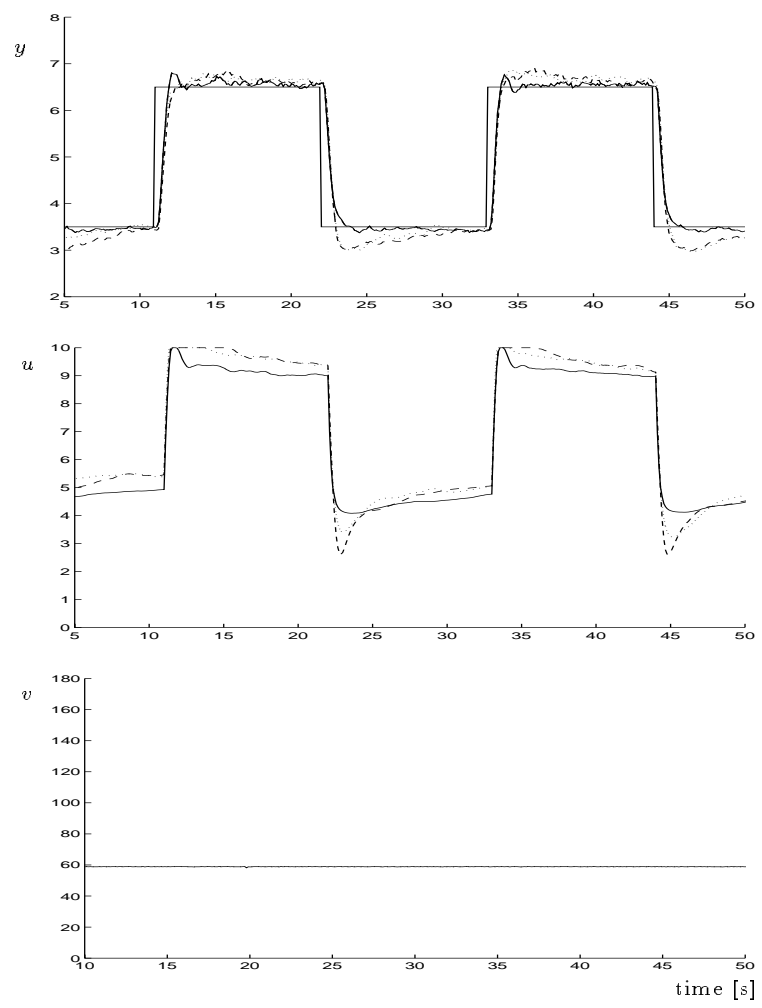

Figure 7: Tracking performance for the 3 controllers. Dashed lines $(---)$ are with the ARX model, solid lines (-) are with the 4-local model, and dotted lines $(\ldots \ldots)$ are with the mechanistic model.

the system. The empirical model is, however, intuitively quite easy to understand. v) The mechanistic model has less parameters than the 4-local model, and the computational complexity may be somewhat larger with the 4-local model.

This example has shown that the modeling framework based on local models and interpolation may give models that are as useful, accurate, and reliable as with mechanistic modeling, even if the system is well understood. Such models may serve as an alternative that may be attractive especially for systems that are not well understood. Moreover, we believe empirical models developed in this framework has significant advantages over many other non-linear empirical modeling frameworks. The reason is that it admits interpretability of the model through the intuitive and easily understandable operating regime concept, and the fact that the local ARX models can be interpreted independently.

\section{Acknowledgments}

This work was supported by the Research Council of Norway under grant no. ST. 10.12.221718 given to the first author. We are grateful to Knut Fjeldheim, who did preliminary experiments in his diploma the-
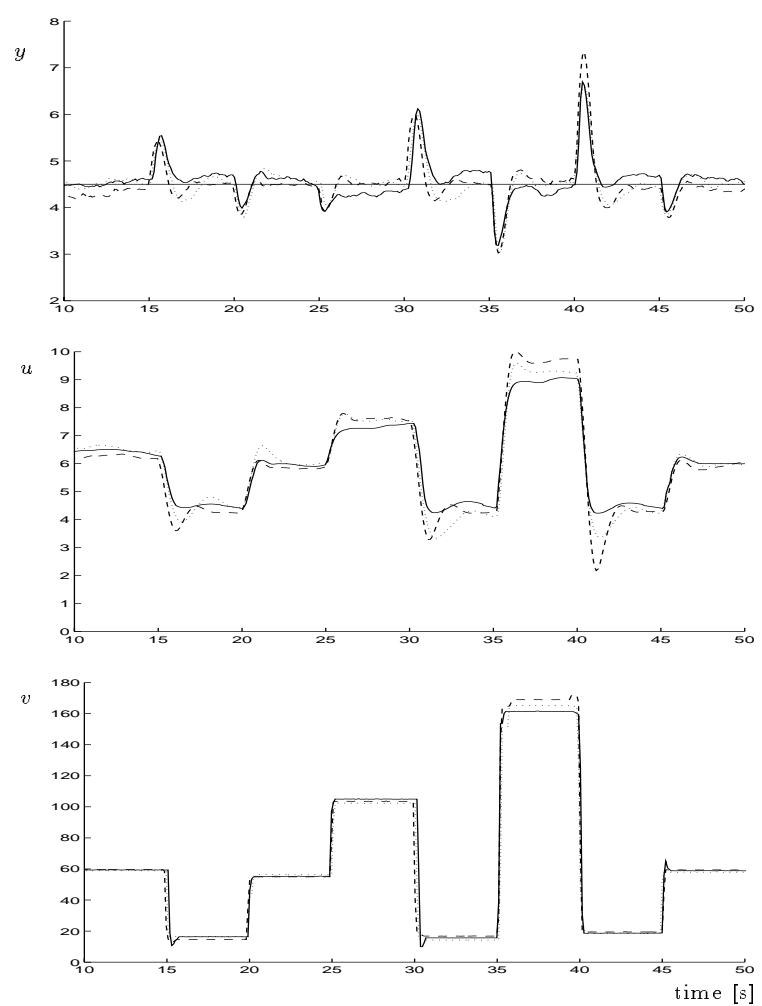

Figure 8: Disturbance rejection performance for the 3 controllers.

sis.

\section{References}

[1] T. Takagi and M. Sugeno. Fuzzy identification of systems and its application to modeling and control. IEEE Trans. Systems, Man, and Cybernetics, $15: 116-132,1985$.

[2] T. A. Johansen and B. A. Foss. Constructing NARMAX models using ARMAX models. Int. $J$. Control, 58:1125-1153, 1993.

[3] B. A. Foss, T. A. Johansen, and Aa. V. Sørensen. Nonlinear predictive control using local models - applied to a batch process. In Preprints IFAC Symp. Advanced Control of Chemical Processes (ADCHEM), Kyoto, Japan (also accepted for Control Engineering Practise), pages 225-230, 1994.

[4] P. Lindskog and L. Ljung. Tools for semiphysical modeling. In Preprints IFAC Symposium on System Identification, Copenhagen, volume 3, pages 237-242, 1994.

[5] P. S. Tuffs and D. W. Clarke. Self-tuning control of offset: A unified approach. IEE Proc. D, 132:100-110, 1985 .

[6] D. W. Clarke, C. Mothadi, and P. S. Tuffs. Generalized predictive control. Automatica, 23:137$160,1987$.

[7] W. H. Press, B. P. Flannery, S. A. Teukolsky, and W. T. Vetterling. Numerical Recipes in C: The Art of Scientific Computing. Cambridge University Press, 1988. 